\title{
Research on Variable Structure Control of Servo System Based on Nonlinear Sliding Mode
}

\author{
Huali $\mathrm{Wu}^{\mathrm{a}}$, Lingling Wang ${ }^{\mathrm{b}}$, Lili Yang, Junwei Lei \\ Department of Control, Naval Aeronautical and Astronautical University, Yantai, 264001, China \\ aemail:tom_linhuali@126.com, bemail: lingling0711@163.com
}

keywords: Servo System; Non-linear; Sliding Mode; Variable Structure

\begin{abstract}
Based on the second-order simplified model of servo system, a new kind of nonlinear sliding mode surface is adopted, and the derivative of sliding surface is derived to obtain the variable structure control law of servo system. The Lyapunov function is chosen to ensure the stability of the whole system and realize the tracking of the desired steering angle. Finally, the simulation results verify the correctness of the proposed method.
\end{abstract}

\section{Introduction}

In the missile and other aircraft control system, the servo system as an executive component, is an important component to complete the heading control, and its performance is particularly significant [1]. In the flight of the aircraft, a number of structural parameters will be dramatically changed, and the time constant and transmission coefficient of the servo will be changed with the changes of servo surface hinge torque and external disturbance torque. Therefore, the control system is required to have good anti-interference performance and robustness. However, the current PID control method does not meet the control requirements of the servo system [2-3]. This paper applies the sliding mode variable structure control algorithm [4-5], which has good control ability for nonlinear system or time-varying linear system, and the sliding mode controller is designed to realize the stability control of the servo system, which is analyzed and verified by simulation results.

\section{Model Description}

A simplified model of a missile servo system can be described as a differential equation 1 [6-7].

$\dot{y}_{a}=-\frac{1}{T} y_{a}+\frac{1}{T} u$

$\dot{y}=y_{a}$

Where $T$ is the inertia time constant, and $T=0.06 \mathrm{~s}, u$ is the control input, $y_{a}$ is the rotation speed of the servo, $y$ is the output rotation angle of the servo, and the relationship between $y_{a}$ and $u$ can be described by the transfer function 2 as follows.

$\frac{y_{a}}{u}=\frac{1}{T S+1}$

Where $S$ is a differential operator in the transfer function.

The whole servo gear system model can be described as figure 1 .

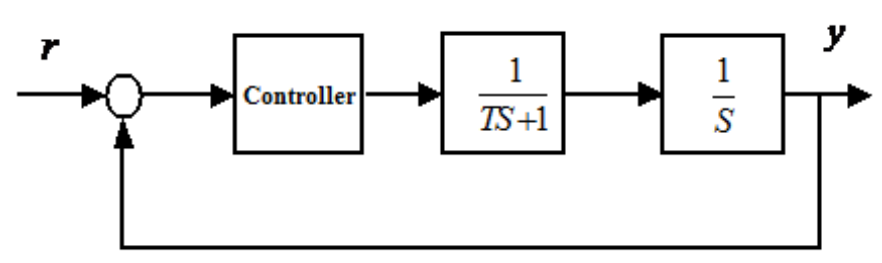

Figure 1 Schematic diagram of servo system 
The control goal is to design the controller, so that the output position $y$ signal of the servo system tracks the desired signal $r$ [8-9].

\section{Design of Nonlinear Sliding Mode Controller}

Define the error variable as $e=r-y$ [10], the sliding surface as formula 3 .

$s=\dot{e}+c_{1} e+c_{2} e^{5 / 3}+c_{3} e^{3}$

Derivation is

$\dot{s}=c_{1} \dot{e}+\ddot{e}+c_{2} \frac{5}{3} e^{2 / 3} \dot{e}+3 c_{3} e^{2} \dot{e}$

Where

$\dot{e}=-y_{a}, \quad \ddot{e}=-\dot{y}_{a}=\frac{1}{T} y_{a}-\frac{1}{T} u$

Substitute formula 4 into the above equation 3 to obtain the formula 6 .

$$
\begin{aligned}
& \dot{s}=\left(c_{1}+c_{2} \frac{5}{3} e^{2 / 3}+3 c_{3} e^{2}\right) \dot{e}+\ddot{e} \\
& =-y_{a}\left(c_{1}+c_{2} \frac{5}{3} e^{2 / 3}+3 c_{3} e^{2}\right)+\left(\frac{1}{T} y_{a}-\frac{1}{T} u\right)
\end{aligned}
$$

Define

$w=-y_{a}\left(c_{1}+c_{2} \frac{5}{3} e^{2 / 3}+3 c_{3} e^{2}\right)+\frac{1}{T} y_{a}$

Then

$\dot{s}=w-\frac{1}{T} u$

The control law can be designed as following formula 9.

$u=T w+T k_{a} s+T k_{b} \operatorname{sgn}(s)$

Then we get formula 10 .

$\dot{s}=-k_{a} s-k_{b} \operatorname{sgn}(s)$

Select Lyapunov function as formula 11.

$V=\frac{1}{2} s^{2}$

Derivation is formular 12.

$\dot{V}=s \dot{s}=-k_{a} s^{2}-k_{b}|s| \leq 0$

From the Lyapunov stability theorem, the system is stable.

\section{Simulation Analysis of Variable Structure Control}

Set the control parameters as follows: $c 1=10, c 2=0.02, c 3=5, k a=20, k b=70$. The simulation results are shown as following figure 2 and figure 3. 


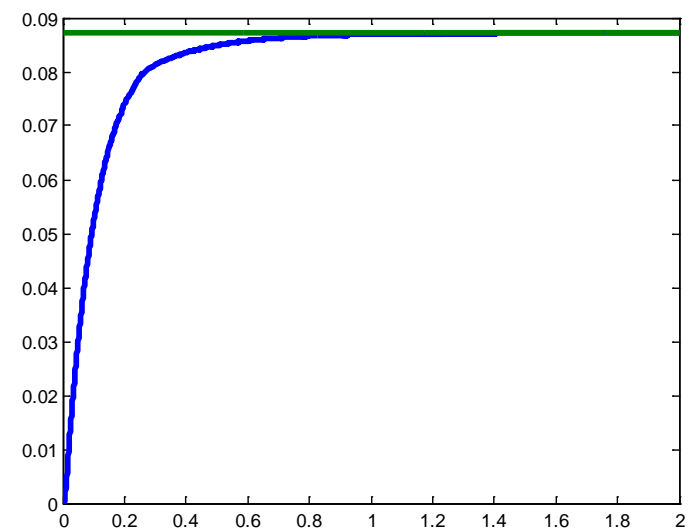

Figure 2 The output response curve of the servo

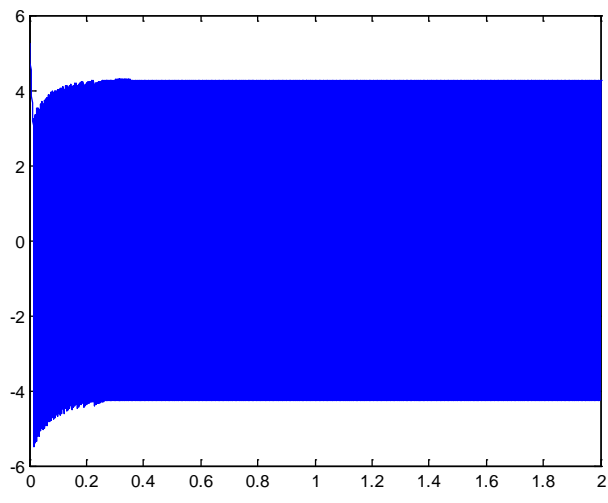

Figure 3 The control volume curve

At this time, the flutter of the control volume is too large, which is not conducive to long-life use of the servo. To reduce the control volume of the symbolic function control, and adjust the control parameters to $\mathrm{c} 1=10, \mathrm{c} 2=0.02, \mathrm{c} 3=5, \mathrm{ka}=20, \mathrm{~kb}=10$, and the simulation results are shown as following figure 4 and figure 5 .

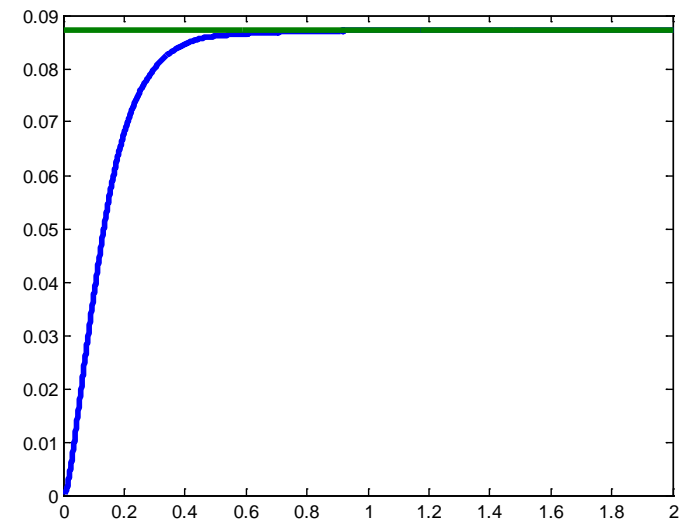

Figure 4 The output response curve of the servo

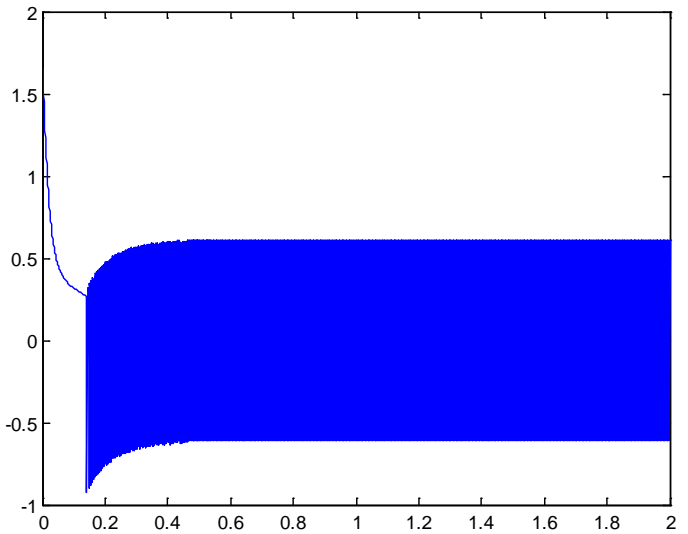

Figure 5 The control volume curve

Adjust the control parameters to $\mathrm{c} 1=10, \mathrm{c} 2=0.02, \mathrm{c} 3=5, \mathrm{ka}=60, \mathrm{~kb}=0.1$, and the simulation results are shown as following figure 6 and figure 7.

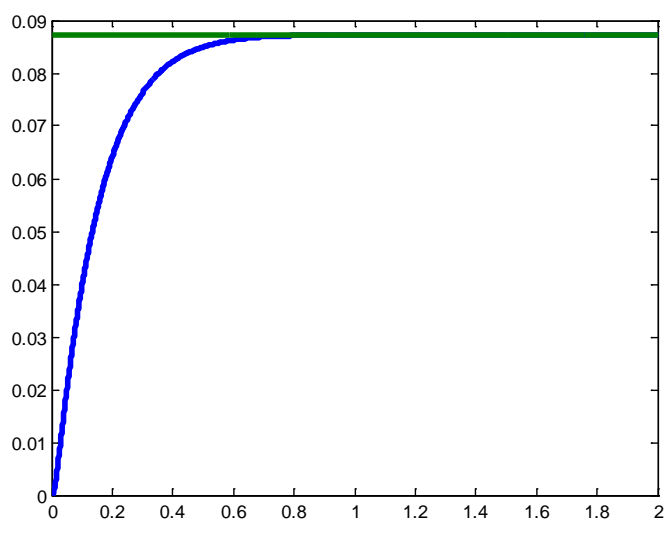

Figure 6 The output response curve of the servo

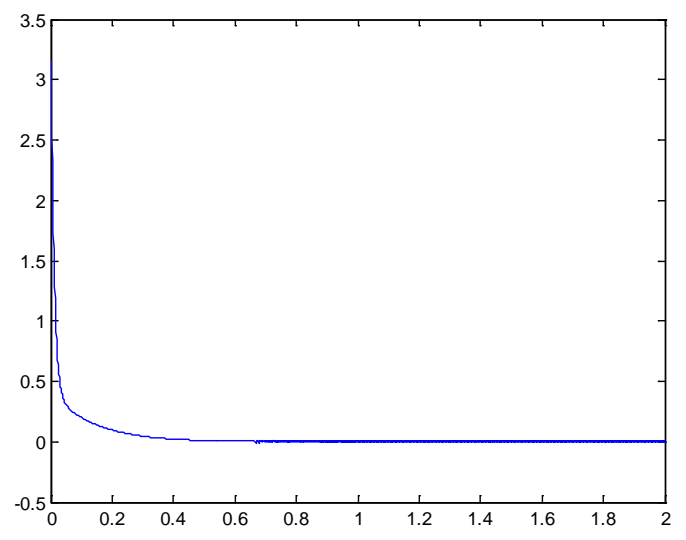

Figure 7 The control volume curve

At this time the system response speed is not fast enough, further adjust the parameters as $\mathrm{c} 1=50$, $\mathrm{c} 2=0.02, \mathrm{c} 3=5, \mathrm{ka}=360, \mathrm{~kb}=0.1$, then the simulation results are as following figure 8 and figure 9. 


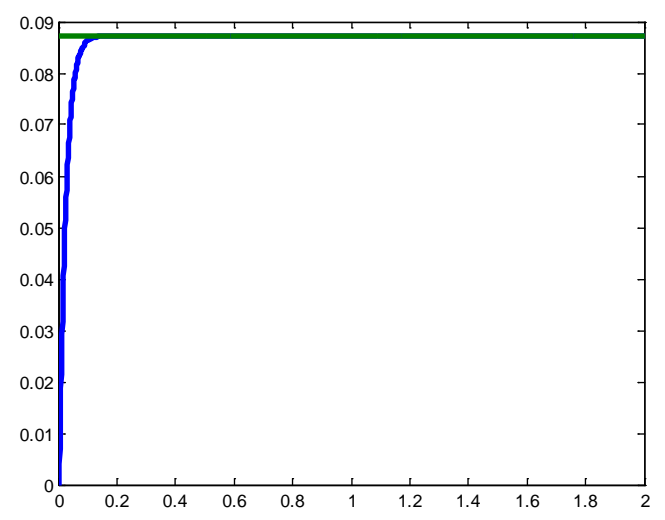

Figure 8 The output response curve of the servo

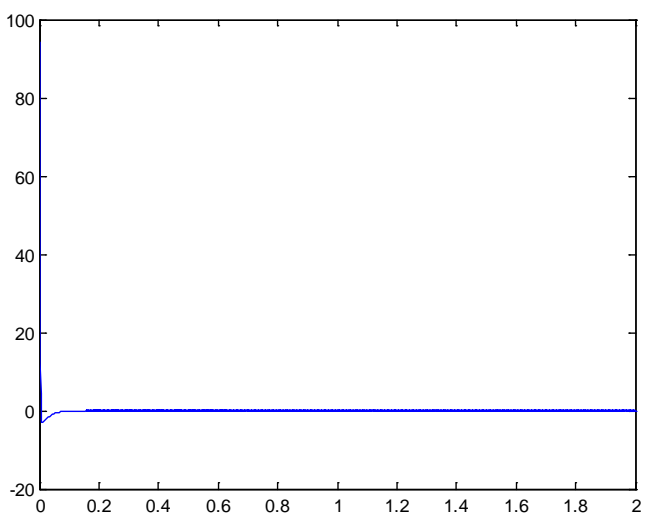

Figure 9 The control volume curve

The simulation results show that the nonlinear sliding mode control algorithm makes the response speed of the servo faster, and the control volume of the servo system is very smooth with no flutter, which meets the engineering needs.

\section{Summary}

Based on the simplified model of the servo system, a nonlinear sliding mode controller satisfying Lyapunov stability is designed and simulated. The simulation results show that the sliding mode variable structure control can achieve the stability control of the servo system, and the response curve is smooth and free of flutter, which can satisfy the requirements of dynamic and steady state performance at the same time.

\section{Reference}

[1] Lin Liu, Sub-system of Flight Control System. National Defense Industry Press, Beijing (2003) (in Chinese)

[2] Teng Zhang, Xiaoyu Ni, Design of control system for civil aircraft steering gear [J], Man-Machine Management and Development, 2012(3):14-17(in Chinese)

[3] Yinze Wang, Xunbo Li, Simulation and Analysis of Servo System of Unmanned Aerial Vehicle Electromechanical Actuator [J], Mechanical Research and Application, 2010(5): 36-38 (in Chinese)

[4] Feng Zhao, Pengchen Gu, Yuhong. Zhao, Application of sliding mode variable structure control in electric steering gear. Shanghai aerospace velocity, 2011, 6(28): 64-68 (in Chinese)

[5] Shuhua Peng, Huade Li, Zhong Su, Sliding Mode Variable Structure Control of Electromechanical Actuator with Uncertain Parameters, Electric Machines and Control, 2009, 1(13): 128-132 (in Chinese)

[6] Jianbo Sun, Shiying Gong, Yahui Dong, Simulation of permanent magnet brushless DC motor speed control system [J], Servo technology, 2001, 2(34): 20-23 (in Chinese)

[7] Man Zeng, Xiaoli Xiong, Wenge Ding, A Design of Typical Digital Brushless Electric Steering Gear [J], Journal of North University of China Natural Science Edition, 2011, 32(6):751-757 (in Chinese)

[8] Yang Chong, Ke Zhang, Jingyu Wang, A Design and Realization of Servo Control Algorithm Based on Fuzzy ADRC [J], Journal of Northwestern Polytechnical University, 2011, 29(2):217-222 (in Chinese)

[9] Can Wang, Ruiqing Ma, Bo Tan, Development of DC Servo Actuator Servo System [J] Micro motor, 2008, 36(10):25-27 (in Chinese) 
[10] Yu Chen, Hongwei Wang, Guoshan Xie, Research on Application of Sliding Mode Variable Structure Control Technology in Ship Autopilot, Marine Electric Technology.2008, 6(28): 351-353 (in Chinese) 